\title{
Research on Innovation and Development of Artificial Intelligence in the Age of Big Data
}

\author{
Zailong Zhou \\ Information center, ShanDong College of Arts, ShanDong College of Arts, Jinan, China \\ randeling@sdca.edu.cn
}

Keywords: big data; artificial intelligence; data mining; robot

\begin{abstract}
Big data and artificial intelligence are two important branches of today's computer science. In recent years, research on two areas of big data and artificial intelligence has been uninterrupted. In fact, the connection between big data and artificial intelligence is inextricably linked. Firstly, the development of big data technology relies on artificial intelligence because it uses many theories and methods of artificial intelligence. Secondly, the development of artificial intelligence must also rely on big data technology and need big data to support it. Under the background of the big data era, what innovations and developments will be made in thefuture of artificial intelligence, we will wait and see.
\end{abstract}

\section{Introduction}

After decades of development of big data and artificial intelligence, the development of artificial intelligence must also rely on big data technology and need big data to support it. Artificial intelligence has made a major breakthrough in the process from theory to practice. Artificial intelligence has the potential to successfully solve various types of problems. With the advent of high-performance computing, artificial intelligence will be more widely used. As artificial intelligence has evolved into a core driver of information and communication technology (ICT) innovation, a large number of companies are scrambling to join the battle for intellectual property. On the one hand, they compete fiercely with each other, and on the other hand, they must be forced to carry out global cooperation. Therefore, the strategic investment and action of artificial intelligence is indispensable for the successful realization of the next generation digital transformation. In the context of big data, artificial intelligence using big data can better facilitate human activities. This paper hopes to help the future of artificial intelligence innovation.

\section{The generation of big data}

In the long history of the development of human history, there has never been a period in which such massive data is generated as it is today, and the generation of such data is not limited by time and space. The generation of human social data has gone through three stages. Each generation has undergone a quantum leap from quantity to quality. It is precisely because of the huge changes in the way data is generated that the concept of big data has emerged.

The earliest database concept emerged to reduce the complexity of data analysis and management, 
and to apply the database to the operational system as its data management system. The first leap in the way human society data was generated was built during the period when the database was widely used. The way this data is generated is passive, with a certain amount of data being generated as the operating system operates and recorded in the database over a period of time. The second leap in data volume changes was based on the Internet, and the Web 2.0 era that followed the rise of the Internet triggered an explosion of data volumes. At this stage, the way data is generated is proactive: a new type of social network that is familiar with people such as blogs and Weibo is gradually emerging, and users will continue to generate large amounts of data when using such applications; using smartphones and tablets as the development of new mobile devices represented by people, people use these mobile devices to publish their opinions online, the way is more convenient, and the data is generated faster. The emergence and widespread application of perceptual systems has brought data into the third phase, while big data is slowly emerging, which is also the root cause of big data. At this stage, the way data is generated is automatic. Intelligent sensors are widely used in various fields such as society, commerce, and science. With the continuous development of integrated circuits, smart sensors will continuously generate new data when they operate.

The emergence of big data will inevitably affect the data source, storage, processing methods and technology issues, bringing changes to the original data management methods. Traditional data sources are relatively small, the amount of data is small, and the data structure is single. The use of relational databases and parallel databases is sufficient to store, analyze, and process data. In the big data environment, the amount of data is large and the types of structures are numerous, and the technical requirements for data storage, analysis, and management are also increased. In addition, the efficiency of processing data and the availability of data cannot be ignored. It is necessary to rely on parallel computing to achieve the above requirements. In the high-speed computing computer, in the analysis and mining of data, the traditional data processing method is processor-centric, and the data will reduce its efficiency during the mobile process. In the big data environment, you need to use a data-centric model to reduce the loss of data movement. On the other hand, the traditional parallel database technology is limited by its information consistency, and the available performance of the data is not guaranteed. Therefore, the traditional data processing methods can't adapt to the high requirements of the era of big data, and it is necessary to evolve new data processing technology theories and methods to cater to the development of the new information age.

\section{The application range of artificial intelligence}

Computer artificial intelligence technology is widely used in various fields, and nowadays there are mainly the following.

\subsection{Application of artificial intelligence technology in medicine}

With the maturity of artificial intelligence technology, artificial intelligence technology has been widely applied to the medical field, and has been widely used in neural network technology, clinical medical diagnosis, and expert systems. For example, on the basis of probabilistic analysis, the medical diagnosis process has been widely applied and achieved remarkable results. In some respects, the medical level and physician professional ability of the hospital can be improved. The application of artificial intelligence technology in the medical industry not only further improves the medical level, but also solves the patient's problems efficiently and accurately.

\subsection{Application of artificial intelligence technology in life}

Face recognition can reach the product level, and Alipay's face success rate exceeds that of 
humans. Nowadays, some mobile phones already have the function of fingerprint unlocking, and it is an application example of artificial intelligence technology in life. Window10 now also has a voice assistant for speech recognition. The United States has applied artificial intelligence technology to micro-cars, so unmanned driving will inevitably be realized in the future.

\subsection{Application of artificial intelligence technology in education}

In the United States, robot assistants have emerged, which can provide guidance to students, promote students' learning, and send e-mails to students in the evening. It is very intimate, which helps to improve students' interest in learning and improve students' learning efficiency. And machine learning that already exists, mainly to study how to implement computer simulation of human learning activities. The use of artificial intelligence technology in learning can improve the learning model and cultivate the various abilities of learners, which is beneficial to the development of quality education. Companies such as Google, Microsoft and Baidu are now doing artificial intelligence translations. People can learn English online. According to research data analysis, the accuracy of these artificial intelligence translations is comparable to the level of human translation. In this way, we can communicate with foreigners freely, and no longer have to worry about language barriers.

\subsection{Application of artificial intelligence technology in military}

In military command systems, the application of artificial intelligence optimizes the use of equipment, and the method used is partly based on calculating the detection range. The application of artificial intelligence also includes the interpretation and classification of signals. Artificial intelligence technology provides a means to introduce the experience of human operators into computer systems, while allowing effective dialogue between users and machines. In the case of sonar classification, the only effective way to solve this complex problem is to rely on automation.

\section{The relationship between big data and artificial intelligence}

In recent years, research in the two fields of big data and artificial intelligence has been mutually promoted, resulting in many new methods, applications and values.

Today, humans have the ability to access, retrieve, classify, and count big data with large data sizes, multiple data types, fast data streams, and high data authenticity, thanks to the development of big data technology. Moreover, some theories and methods in the field of artificial intelligence have begun to be used in big data analysis and have achieved certain results. The study found that solving the problem of the expansion and growth of artificial intelligence is inseparable from big data technology.

In the past, artificial intelligence technology could not achieve the same learning and research capabilities as humans. The reason is that artificial intelligence seems to be simple, it is actually a very cumbersome and complicated thing. The two necessary conditions for generating artificial intelligence are the support of massive data and the strong processing ability of these data, while previous machines did not have these two conditions.

Artificial intelligence, like humans, requires a lot of knowledge and rich experience. Behind this knowledge and experience is the need for a large amount of data support. The further development of big data technology provides a certain technical support for storing and analyzing a large amount of data, so that the amount of data obtained by the machine and the data processing capability possessed by the machine are matched with the amount of data and data processing capability required to form artificial intelligence. Only in this way can artificial intelligence be developed. The development of 
artificial intelligence, in turn, further promotes the forward development of big data technology and forms an effective mutual promotion.

Rather than relying on big data for the development of artificial intelligence, it is better to say that big data opens a new chapter in artificial intelligence. Some theories and methods in the field of artificial intelligence can effectively enhance the use value of big data. At the same time, the development of big data technology will also provide a place for artificial intelligence.

\section{Future development of artificial intelligence}

With the continuous development of big data technology and computer science and technology, the future development of artificial intelligence will mainly be in the following aspects: pattern recognition, expert system, symbolic calculation, artificial neural network and machine emotion.

\subsection{Pattern recognition}

Pattern recognition, as the name implies, refers to the use of mathematical calculation methods to study the automatic interpretation and processing of patterns. It can be concluded that with the continuous development of computer technology, human beings will be able to conduct further in-depth research on complex information processing. At the same time, the pattern recognition function also creates feasible clues and provides necessary assistance for human beings to recognize their own intelligence.

\subsection{Expert system}

The expert system is an important direction for the development of artificial intelligence in the future. Expert systems have been widely used in today's life. In fact, the expert system refers to a program system with a large amount of specialized knowledge and experience in the industry or field. It is mainly based on computer science technology and artificial intelligence technology. It firstly conducts in-depth reasoning and judgment based on some relevant knowledge and relevant experience provided by some authoritative experts or experts in a certain industry or field, and then can simulate human experts that determine the decision process. Through this process, we help people solve some of the complex problems that need to be dealt with by human experts in reality.

\subsection{Symbolic calculation}

Scientific computing is one of the most basic and primary uses since the invention of the computer. Scientific calculations can be divided into two categories: one is the calculation of pure values, and the other is symbolic calculation. Symbolic calculation is different from traditional pure numerical calculation. It is an intelligent calculation, which is mainly calculated by processing corresponding symbols. In symbolic calculations, symbols can represent very many types, such as real numbers, complex numbers, integers, rational numbers, etc. Symbols can also be used to represent functions, polynomials, sets, etc.

\subsection{Artificial neural network and machine emotion}

The development of computer technology to this day, the basic idea of artificial intelligence has been applied in many fields. One of the most important new areas of future artificial intelligence applications is the artificial neural network. Studies have shown that emotions are part of intelligence and not separate from intelligence. Therefore, it can be asserted that the next breakthrough in the 
future development of artificial intelligence is to give computer emotional ability and emotional intelligence.

\section{Summary}

The application of artificial intelligence in the future will create more and more advanced intelligent "products" for human beings to serve human beings, and artificial intelligence will surpass human intelligence in more and more fields. Under the background of the big data era, I believe that artificial intelligence will be greatly developed, and more discoveries, inventions and achievements will appear in front of everyone. As if you can see, the intelligence that is the same as human level and even surpasses human beings is about to be realized. I believe that this moment is in the near future, let everyone wait and see.

\section{References}

[1] Jin C Y, Jin L I, Yan L I. The Application of Artificial Intelligence to System Engineering of Innovation Laboratory Management [J]. Research \& Exploration in Laboratory, 2010.

[2] Zhong Y X. Breakthroughs in artificial intelligence and innovation in methodology[J]. Pattern Recognition \& Artificial Intelligence, 2012, 25(3):456-461.

[3] Meert W, Nys V, Theunis R, et al. Innovation Lab @ KU Leuven: Education, Engineering and Artificial Intelligence[C]// 2015.

[4] Jin C Y, Jin L I, Yan L I. The Application of Artificial Intelligence to System Engineering of Innovation Laboratory Management[J]. Research \& Exploration in Laboratory, 2010.

[5] Hendler J, Subramanian D, Uthurusamy R, et al. Proceedings of the sixteenth national conference on Artificial intelligence and the eleventh Innovative applications of artificial intelligence conference innovative applications of artificial intelligence[J].

[6] Yun J H, Lee D, Ahn H, et al. Not Deep Learning but Autonomous Learning of Open Innovation for Sustainable Artificial Intelligence[J]. Sustainability, 2016, 8(8):797.

[7] Briceño J, Cruz-Ramírez M, Prieto M, et al. Use of artificial intelligence as an innovative donor-recipient matching model for liver transplantation: Results from a multicenter Spanish study[J]. Journal of Hepatology, 2014, 61(5):1020-8.

[8] Zhou X. Artificial intelligence strategy interpretation: strategy for American innovation[J]. Telecommunications Network Technology, 2017.

[9] Poblet M, Casanovas P, Plaza E. Linked Democracy: Artificial Intelligence for Democratic Innovation Proceedings IJCAI 2017 Workshop[C]// Linked Democracy: Artificial Intelligence for Democratic Innovation. 2017.

[10] Analysis on Service Pattern and Innovation Development of Library_from the Perspective of Artificial Intelligence: Interpretation of the Report Entitled "Artificial Intelligence: Opportunities and Implications for the Future of Decision Making”[J]. Library \& Information, 2017.

[11] Liu K, Xiangen H U, Wang P, et al. Do Machines Need Education? A Review on the Education of General Artificial Intelligence and Educational Innovation[J]. Open Education Research, 2018.

[12] Wang Q, Wang C Y. Discussion and Practice on Innovation of Undergraduate Artificial Intelligence Course[J]. Value Engineering, 2017.

[13] Yagi T, Ozawa H. Creation of artificial intelligence services through open innovation[J]. Ntt Technical Review, 2017, 15(8).

[14] Cockburn I M, Henderson R, Stern S. The Impact of Artificial Intelligence on Innovation[J]. Nber Chapters, 2018. 